\title{
Equivalence Checking for Superconducting RSFQ Logic Circuits*
}

\author{
Rongliang Fu ${ }^{\dagger \dagger}$, Junying Huang ${ }^{\dagger}$, Zhi-Min Zhang ${ }^{\dagger}$ \\ ${ }^{\dagger}$ State Key Laboratory of Computer Architecture, Institute of Computing Technology, Chinese Academy of Sciences \\ ${ }^{\ddagger}$ School of Computer Science and Technology, University of Chinese Academy of Sciences \\ Beijing, China \\ zzm@ict.ac.cn
}

\begin{abstract}
Equivalence checking is a key component of the verification methodology for digital circuit designs. In this paper, we propose an equivalence checking framework for superconducting rapid single-flux-quantum (RSFQ) logic circuits which include acyclic circuits and bit-slice-based cyclic circuits. It consists of a structure checker and a logic checker. The structure checker is used to check whether the circuit meets the design rules of superconducting RSFQ logic circuits. The logic checker can be used to check whether two RSFQ gate-level circuits have the same logic function. For the logic checker, we propose a logic equivalence checking method based on logic cone partition. The circuit network is simplified layer by layer and iteratively partitioned into logic cones, each of which is verified by the SMT solver. The experimental results show the feasibility of our approach on superconducting RSFQ logic circuits.
\end{abstract}

\section{CCS CONCEPTS}

- Hardware Emerging technologies Superconducting circuits - Hardware Equivalence checking • Hardware Theorem proving and SAT solving

KEYWORDS: Superconducting circuits, Rapid single-fluxquantum (RSFQ), Equivalence checking, Logic cone, SMT

\section{ACM Reference format:}

Rongliang Fu, Junying Huang and Zhi-Min Zhang. 2021. Equivalence Checking for Superconducting RSFQ Logic Circuits. In Proceedings of 2021 Great Lakes Symposium on VLSI (GLSVLSI'21). ACM, Virtual Event, USA. ACM, New York, NY, USA. 6 pages. https://doi.org/10.1145/3453688.3461486

Permission to make digital or hard copies of all or part of this work for personal or classroom use is granted without fee provided that copies are not made or distributed for profit or commercial advantage and that copies bear this notice and the full citation on the first page. Copyrights for components of this work owned by others than the author(s) must be honored. Abstracting with credit is permitted. To copy otherwise, or republish, to post on servers or to redistribute to lists, requires prior specific permission and/or a fee. Request permissions from Permissions@acm.org. GLSVLSI'21, June 22-25, 2021, Virtual Event, USA.

(C) 2021 Association for Computing Machinery.

ACM ISBN 978-1-4503-8393-6/21/06...\$15.00.

DOI: https://doi.org/10.1145/3453688.3461486

\section{Introduction}

Low-temperature superconductivity is a very peculiar macroscopic quantum phenomenon wherein certain materials have zero electrical resistance characteristics at very low absolute temperatures. Compared with transistor-based complementary metal-oxide-semiconductor (CMOS) computing systems, computing systems built with some superconducting materials can provide orders of magnitude higher speed and higher energy efficiency, such as superconducting single-flux-quantum (SFQ) logic circuits.

SFQ-based logic circuits use Josephson junctions as the active components which have a rapid switching speed (a few picosecond/bit) and low switching energy $\left(10^{-19} \mathrm{~J} / \mathrm{bit}\right)$ at around 4 $\mathrm{K}$ [1]. As a member of the SFQ family, superconducting rapid single-flux-quantum (RSFQ) technology currently has significant advancements from device fabrication and architecture [2]. It has been proved that RSFQ-based T-type flip-flops can work normally at operating frequencies up to $770 \mathrm{GHz}$ [3].

Equivalence checking is a crucial task in VLSI CAD, especially the checking based on functional equivalence. For CMOS circuits, there are already some mature tools that can solve this problem. However, these tools cannot be directly applied to RSFQ circuits due to the following significant distinctions between RSFQ circuits and CMOS circuits.

1. In superconducting RSFQ logic circuits, the logic information is presented in the form of single-flux-quantum pulses, which are very short voltage pulses [4], while CMOS circuits use DC voltage levels to represent the logic information.

2. Almost all RSFQ logic cells have a clock input. The D-type flip-flops (DFFs) should be inserted into the netlist to ensure path balancing. That is, for each gate in the RSFQ circuit, the number of clocked gates in any path from any primary input (PI) to it is the same. Therefore, RSFQ circuits have a natural gate-level pipelined architecture.

3. Each logic cell can only drive one logic cell. To solve the multi-fan-out problem, RSFQ circuits use a splitter to generate two or three output pulses after a certain delay. 


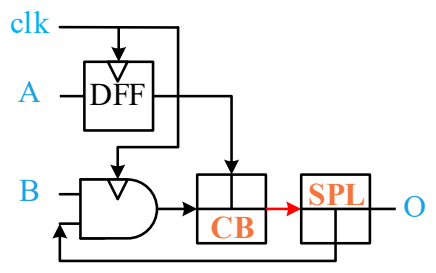

Figure 1: The schematic of the bit-slice-based loop.

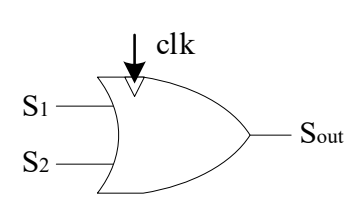

(a)

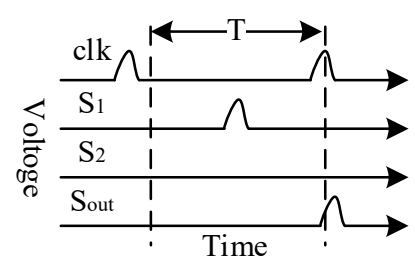

(b)
Figure 2: An OR in RSFQ circuits. (a) The schematic. (b) Signal consequence.

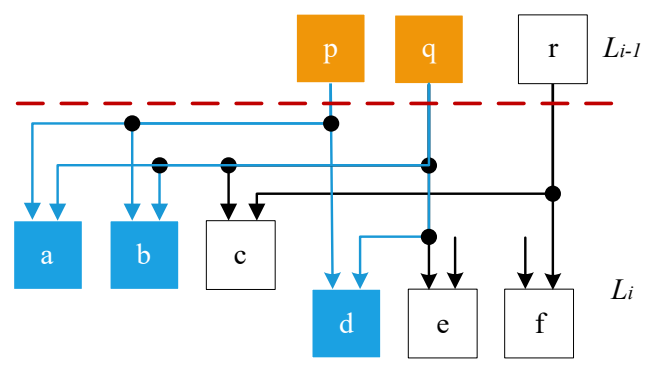

Figure 3: The schematic of finding equivalent vertices.

Although some simulators such as JoSim [5] can be used to verify RSFQ circuits, they are not suitable for large-scale circuits. To address this problem, some verification tools for RSFQ circuits have been proposed, such as VeriSFQ [6] and qEC [7]. They provide structure checkers to check whether the acyclic SFQ circuit meets the fan-out restriction and the path-balanced requirement. However, they mainly concentrate on the acyclic SFQ circuits, and are not suitable for cyclic circuits, for instance, the bit-slice-based cyclic circuits [8]. As shown in Figure 1, the feedback loops in the bit-slice-based cyclic circuits include no clocked gate, making the pipeline processing of slices continuous. As the most important component of the microprocessor, several bit-slice-based RSFQ ALUs have been designed [9]. And a 4-bit bitslice-based ALU for a 32-bit RSFQ microprocessor was demonstrated at $50 \mathrm{GHz}$ successfully [10]. Therefore, an equivalence checking framework for bit-slice-based cyclic circuits is an absolute requirement.

In this paper, we propose an equivalence checking framework for superconducting RSFQ logic circuits including acyclic circuits and bit-slice-based cyclic circuits, as shown in Figure 4. It can check whether any net of the circuit has only two pins and whether the circuit is completely path-balanced as a gate-level pipeline. Besides, it can also verify whether two gate-level RSFQ

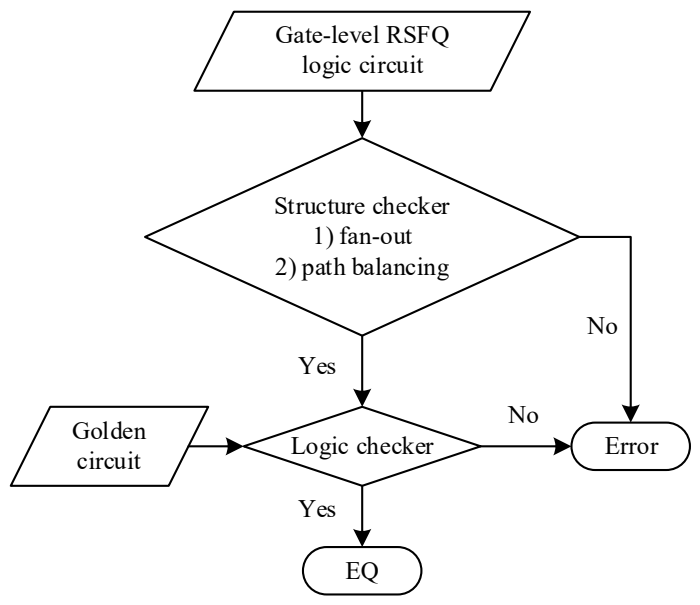

Figure 4: The flow of the equivalence checking framework.

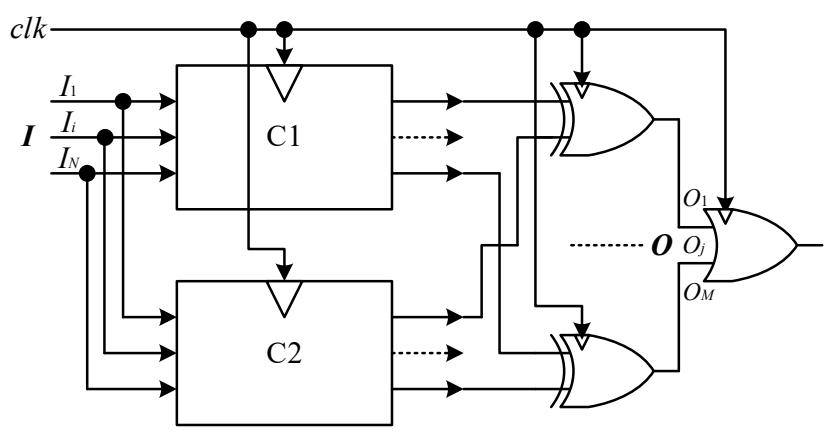

Figure 5: The miter constructed by RSFQ circuits $C_{1}$ and $C_{2}$.

circuits have the same logic function. The contributions of this paper can be summarized in the following:

1. We first propose the equivalence checking framework for superconducting RSFQ logic circuits including acyclic circuits and bit-slice-based cyclic circuits. The framework consists of a structure checker and a logic checker.

2. We propose an approach to determine whether the circuit is path-balanced. This method is suitable for acyclic circuits and bit-slice-based cyclic circuits.

3. We propose a logic equivalence checking method based on logic cone partition. First, a miter is constructed by connecting outputs of two circuits using an "exclusive-or" (XOR) cell, as shown in Figure 5. Then all vertices in the miter network are layered according to the logic level [11], and the equivalent vertices are found and merged layer by layer. Finally, the miter network is iteratively divided into logic cones, each of which is verified by the SMT solver. Compared with $\mathrm{ABC}$, the efficiency of our method is greatly improved.

4. We equate the bit-slice-based loop as a flip-flop, and then verify bit-slice-based cyclic circuits with the equivalence checking tool of CMOS sequential circuits. 
The rest of the paper is organized as follows. Section 2 introduces how to calculate the logic level and determine whether the circuit meets the fan-out restriction and pathbalanced requirements. Section 3 focuses on how to simplify and divide the miter network, and how to use the SMT solver to verify the logic cone, and finally verify the miter network. Section 4 shows experimental results proving the feasibility of our approach on the superconducting RSFQ benchmark. Finally, the conclusions are summarized in Section 5.

\section{Structure checker}

Due to the characteristics of superconducting RSFQ logic circuits mentioned in Section 1, RSFQ circuits need to satisfy the fan-out restriction and the path-balanced requirement. The circuit network is constructed from a gate-level netlist. The structure checker determines whether the circuit meets the fan-out restriction by checking whether each net of the netlist has only two pins. The structure checker determines whether the circuit meets the path-balanced requirement by checking whether any input of the gate in the netlist has the same logic level, that is the maximum number of clocked gates in the path from any primary input to the gate.

In RSFQ bit-slice-based microprocessors, feedback loops are required in the data path for the "carry" signal in bit-slice-based cyclic circuits. The bit-slice-based loop consists of an AND, a CB, a splitter, as shown in Figure 1. For the path balancing checking of bit-slice-based cyclic circuits, we have to deal with the bitslice-based loops. Before calculating the logic level, the bit-slicebased loop needs to be broken by reversing the edge between the $\mathrm{CB}$ and the splitter in the bit-slice-based loop, as shown in Algorithm 1 with linear time complexity of $\mathrm{O}(|E|)$. After removing all sinks and sources (line 3 - 8), the remaining vertices are in the loops. Reversing the edge between CB and splitter can eliminate the bit-slice-based loop (line 10 - 12).

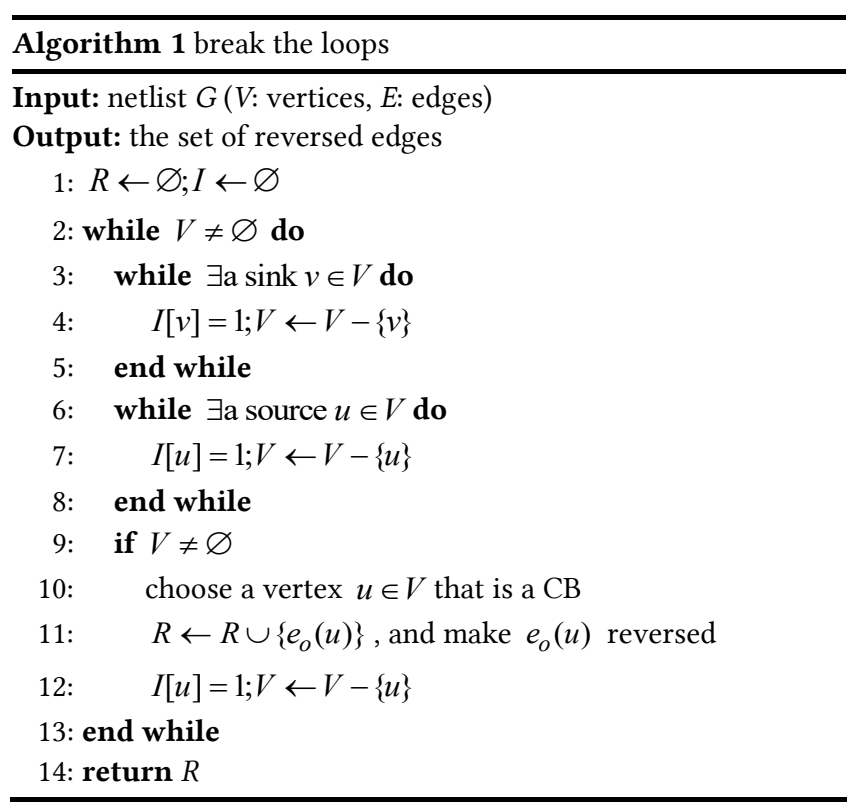

The edge is shown in the red line in Figure 1.

The structure checker uses Algorithm 2 to calculate the logic level and determine whether the circuit is path-balanced. In Algorithm 2, we first use breadth-first search to traverse the circuit network from the primary inputs to the primary outputs and calculate the logic level of each gate (line 3 - 10). For an edge $(v, u)$, the logic level $L[u]$ of the gate $u$ is calculated as

$$
L[u]= \begin{cases}\lfloor L[v]\rfloor+1, & u \text { is a clockd gate or PO } \\ L[v]+1 /|V|, & \text { others }\end{cases}
$$

If there are loops in the circuit network, there are some gates that have not been visited, so we then use breadth-first search to traverse the circuit network from the primary output to the primary input and calculate the logic level of each gate again (line $12-23)$. For an edge $(v, u)$, the logic level $L[v]$ of the gate $v$ is calculated as

$$
L[v]= \begin{cases}0, & v \text { is a PI } \\ \lfloor L[u]\rfloor-1, & v \text { and } u \text { are clockd gates } \\ \lfloor L[u]\rfloor, & v \text { is a clockd gate } \\ L[u]-1 /|V|, & \text { others }\end{cases}
$$

Besides, to make the splitter closer to its successors, the logic level of the splitter is updated according to the minimum logic level of its successors (line 14). For a vertex $v$, the logic level

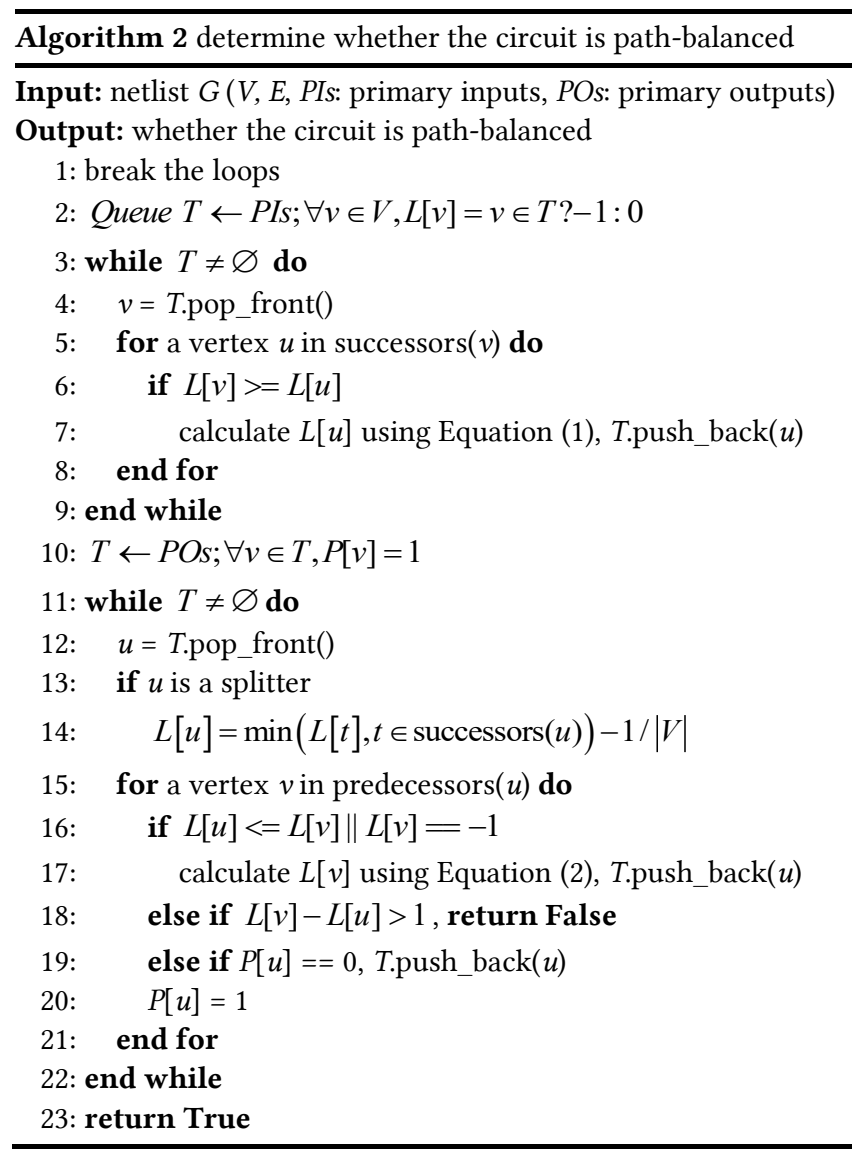


difference between $v$ and any of its predecessors is greater than 1 , the circuit does not meet the path-balanced requirements (line 19). Algorithm 2 has a linear time complexity of $\mathrm{O}(|E|)$.

\section{Logic checker}

In superconducting RSFQ logic circuits, the value of the signal can be represented by whether an SFQ pulse arrives in the clock cycle. In Figure 2, an SFQ pulse arrives at the pin $S_{1}$ in the current clock period, the value of the signal $S_{1}$ is the binary " 1 ". the value of the signal $S_{2}$ is the binary " 0 " due to the absence of the SFQ pulse in this period. When the clock pulse clk arrives, the output pulse $S_{\text {out }}$ is developed, whose value is predetermined by the input signal pulses in the current clock period. So, an RSFQ logic cell is equivalent to the combination of an asynchronous logic circuit and a register [4]. The register stores output signals of the RSFQ logic cell until the clock pulse arrives. Furthermore, the clock pulse can reset the RSFQ logic cell, which makes the next clock period unaffected by the current clock period. Therefore, for SFQ circuits that only contain logic cells such as AND, OR, XOR, etc., the clock signal only drives the output of the clocked gate and does not affect the output value.

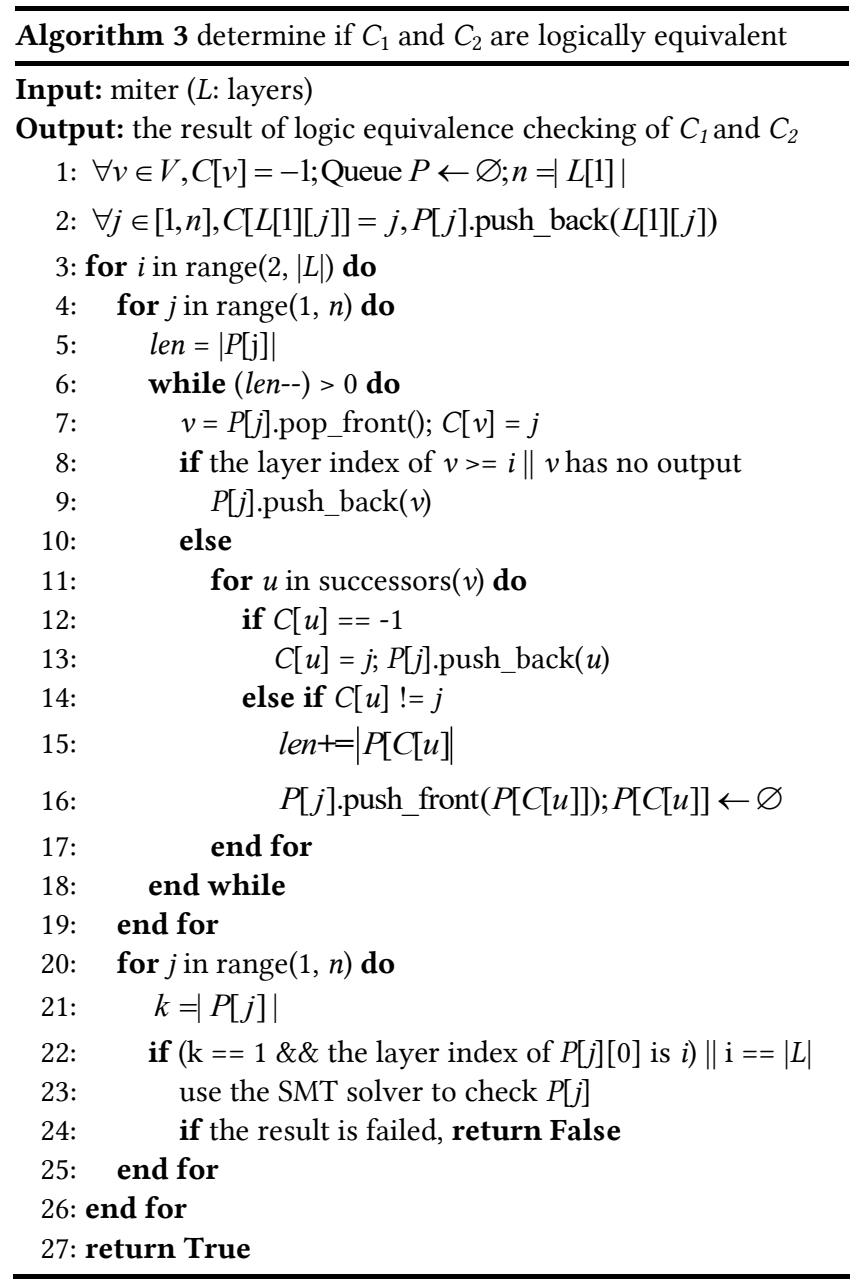

The process of logic equivalence checking does not need to consider the influence of the clock signal of the clocked gate, which makes it equivalent to combinational equivalence checking of CMOS circuits.

For an RSFQ circuit $C$ with $N$ primary inputs $\left(P I_{1}, P I_{2}, \ldots, P I_{i}\right.$, $\left.\ldots, P I_{N}\right)$ and $M$ primary outputs $\left(P O_{1}, P O_{2}, \ldots, P O_{j}, \ldots, P O_{M}\right)$, the value range of $P I_{i}$ and $P O_{j}$ is 0 or 1 . Circuit $C$ can be expressed as a Boolean function $f_{C}:\{0,1\}^{N} \rightarrow\{0,1\}^{M}$. To prove whether the two circuits $C_{1}, C_{2}$ are logically equivalent, we only need to determine whether the outputs of $C_{1}$ and $C_{2}$ are the same for each group of inputs.

$$
\left(C_{1} \Leftrightarrow C_{2}\right) \Leftrightarrow \forall P I \in\{0,1\}^{N}, \sum_{j=1}^{M} P O_{1, j} \oplus P O_{2, j} \equiv 0
$$

For two RSFQ circuits $C_{1}$ and $C_{2}$ to be verified, the pairs of primary inputs with the same names are connected, and each corresponding pair of primary outputs is connected to an XOR gate, and these XOR gates constitute the outputs of the miter. Furthermore, we can also feed all outputs of these XOR gates into an OR gate and use the output of this OR gate as the only output of the miter, so that if and only if $C_{1}$ and $C_{2}$ are logically equivalent, the output of the miter is a constant 0 . As shown in Figure 5 , the inputs of miter are denoted as $I$, and the outputs are denoted as $O$.

$$
\begin{aligned}
& \left\{\begin{array}{l}
I_{i}=P I_{1, i}=P I_{2, i} \\
O_{j}=P O_{1, j} \oplus P O_{2, j}
\end{array} \quad i \in[1, N], j \in[1, M]\right. \\
& \left(C_{1} \Leftrightarrow C_{2}\right) \Leftrightarrow \forall I \in\{0,1\}^{N}, \sum_{j=1}^{M} O_{j} \equiv 0
\end{aligned}
$$

The miter constructed above is usually a very large network containing a large number of redundant and repeated vertices. The simplification of the miter plays a very important role in logic equivalence checking. In superconducting RSFQ logic circuits, the circuit has a natural gate-level pipelined architecture. The miter network can be layered according to the logic level. Let $L$ be the set of layers after miter layered. The first layer $L_{1}$ is the set of all inputs of the miter, and the last layer $L_{|L|}$ is the set of all outputs of the miter. We merge the equivalent vertices layer by layer from $L_{1}$ to $L_{|L|}$ with linear time complexity. For a vertex $v$ in $L_{i}$, let $P$ is the set of its predecessors, and $S_{k}$ is the set of all successors of the predecessor $P_{k}$. The set of equivalent vertices of vertex $v$ :

TABLE I. THE TRUTH TABLE OF ANDF

\begin{tabular}{|c|c|c|c|}
\hline $\mathrm{A}$ & $\mathrm{B}$ & $\mathrm{O}(\mathrm{n})$ & $\mathrm{O}(\mathrm{n}+1)$ \\
\hline 0 & 0 & 0 & 0 \\
\hline 0 & 0 & 1 & 0 \\
\hline 0 & 1 & 0 & 0 \\
\hline$*$ & 1 & 1 & 1 \\
\hline 1 & $*$ & ${ }^{*}$ & 1 \\
\hline
\end{tabular}




$$
\left.E=\bigcap_{k=1}^{|P|} \quad\left(P_{k}\right)\right\}-\{v\}
$$

In Figure 3, for vertex $a$ in layer $L_{i}$, we first find the set $P=\{p, q\}$ of its predecessors, and then respectively find the set $S_{p}=\{a, b, d\}$ and $S_{q}=\{a, b, c, d, e\}$ of all successors of the predecessor $p$ and $q$. The set of equivalent vertices of vertex $a$ is $E=S_{p} \cap S_{q}-\{a\}=\{b, d\}$. After obtaining all the equivalent vertices of vertex $a$, all outputs of each equivalent vertex $e$ are merged into the output set of vertex $a$, and then vertex $e$ is deleted, thus completing the merging of equivalent vertices.

After simplification, the miter needs to be divided into logic cones iteratively, each of which can be verified by the SMT solver. The logic cone is in the shape of a pyramid. The in-degree of all vertices $I_{c}$ at the bottom of the tower is 0 , and there is only one vertex $O_{c}$ at the top of the tower, and the logic cone includes all nodes in any path from the top of the tower to the bottom of the tower. Algorithm 3 describes the process of logical cone division and verification. We initialize the number of logic cones with the number of primary inputs and use the method of graph coloring to divide the logic cones layer by layer in ascending order of logic level (line 4 -19). For the verification of the logic cone, we convert it into a linear equation $f_{\text {cone }}\left(I_{c}\right)=O_{c}$ and use the SMT solver to solve it (line $22-24$ ). When $O_{c}$ is a primary output, to prove that $O_{c}$ is a constant 0 , we turn this problem into another problem, that is, for $O_{c}=1$, whether there is a solution $I_{c}$ for the equations $f_{c}\left(I_{c}\right)=1$. If the solution exists, $C_{1}$ and $C_{2}$ are not logically equivalent, otherwise, they are logically equivalent at vertex $O_{c}$. When $O_{c}$ is not a primary output, if

TABLE II. EXPERIMENTAL RESULTS OF STRUCTURE CHECKER

\begin{tabular}{|c|c|c|c|}
\hline Circuits & cell & Error & runtime (s) \\
\hline \multirow{2}{*}{ c1908 } & 1475 & No & 0.0160 \\
\cline { 2 - 4 } & 1473 & Yes & 0.0102 \\
\hline \multirow{2}{*}{ c3540 } & 3863 & No & 0.0358 \\
\cline { 2 - 4 } & 3861 & Yes & 0.0235 \\
\hline \multirow{2}{*}{ c432 } & 1542 & No & 0.0147 \\
\cline { 2 - 4 } & 1540 & Yes & 0.0098 \\
\hline \multirow{2}{*}{ c5315 } & 794 & No & 0.0070 \\
\cline { 2 - 4 } & 792 & Yes & 0.0065 \\
\cline { 2 - 4 } C6288 & 8220 & No & 0.0842 \\
\cline { 2 - 4 } & 1218 & Yes & 0.0796 \\
\hline \multirow{2}{*}{ c7552 } & 10992 & No & 0.1177 \\
\cline { 2 - 4 } & 8349 & Yes & 0.1028 \\
\hline \multirow{2}{*}{ c880 } & 8347 & No & 0.0894 \\
\cline { 2 - 4 } & 2110 & Yes & 0.0853 \\
\hline \multirow{2}{*}{ 1-bit ALU } & 2108 & Yes & 0.0189 \\
\cline { 2 - 4 } & 46 & No & 0.0165 \\
\hline \multirow{2}{*}{ 2-bit ALU } & 44 & Yes & 0.0007 \\
\cline { 2 - 4 } & 86 & No & 0.0006 \\
\hline \multirow{2}{*}{ 4-bit ALU } & 160 & Yes & 0.0010 \\
\cline { 2 - 4 } & 158 & No & 0.0008 \\
\hline
\end{tabular}

$f_{c}\left(I_{c}\right) \equiv 1$, let vertex $O_{c}$ be a constant 1 ; if $f_{c}\left(I_{c}\right) \equiv 0$, let vertex $O_{c}$ be a constant 0 ; otherwise, let vertex $O_{c}$ be a primary input. The division and verification process of the logic cone does not end until all nodes are traversed. Algorithm 3 has a linear time complexity of $\mathrm{O}(|E|)$.

For bit-slice-based cyclic circuits, we equate the bit-slicebased loop as a flip-flop called ANDF. And we use the sequential equivalence checking tool of CMOS circuits to check whether the bit-slice-based cyclic circuits are logically equivalent. The characteristic equation for ANDF can be given as:

$$
O(n+1)=A+B^{*} O(n)
$$

The truth table of ANDF is shown in TABLE I.

\section{Experimental results}

The Experiments were performed in Debian 10 (buster) installed in VMWare 15 Pro with a $2.60 \mathrm{GHz}$ Intel Core i7-6700HQ processor with 4 GB memory. The time unit is second (s). The proposed framework has been implemented using $\mathrm{C}++$ language. Boolector [12] is used as an SMT solver. The RSFQ logic gatelevel circuits are composed of two parts, one part is generated by ISCAS85 benchmark circuits [13] using the logic synthesis tool [14], and the other part contains several bit-slice-based ALU circuits [8]. For different types of errors, the result of each case is the average of 10 runs of the program.

For evaluating our structure checker, we insert some structural errors into the netlist by randomly deleting splitters or DFFs. The results are shown in TABLE II. The second column represents the total number of logic gates in the circuit. The third column represents the result of the structure checker.

For evaluating our logic checker, we use the "dc2" and "resyn2" commands of the $\mathrm{ABC}$ tool to pre-synthesize each circuit in the ISCAS85 benchmark to obtain a pair (golden,

\begin{tabular}{|c|c|c|c|c|c|c|c|}
\hline \multirow{2}{*}{ Circuits } & \multicolumn{2}{|c|}{ cell } & \multirow{2}{*}{ result } & \multicolumn{2}{|c|}{ Our method } & \multirow{2}{*}{$\frac{\mathrm{ABC}}{\text { runtime (s) }}$} & \multirow{2}{*}{ reduce $(\%)$} \\
\hline & golden & revised & & merged & runtime (s) & & \\
\hline \multirow{2}{*}{ c1908 } & \multirow{2}{*}{1475} & \multirow{2}{*}{1591} & EQ & 662 & 0.0391 & 0.1382 & $72 \%$ \\
\hline & & & NEQ & 634 & 0.0056 & 0.0221 & $75 \%$ \\
\hline \multirow{2}{*}{ c3540 } & \multirow{2}{*}{3863} & \multirow{2}{*}{3624} & $\mathrm{EQ}$ & 2011 & 0.2484 & 0.5327 & $53 \%$ \\
\hline & & & NEQ & 1982 & 0.0088 & 0.0298 & $71 \%$ \\
\hline \multirow{2}{*}{ c432 } & \multirow{2}{*}{1542} & \multirow{2}{*}{1571} & EQ & 1022 & 0.0255 & 0.0382 & $33 \%$ \\
\hline & & & NEQ & 1022 & 0.0032 & 0.0000 & 0 \\
\hline \multirow{2}{*}{ c499 } & \multirow{2}{*}{794} & \multirow{2}{*}{733} & EQ & 470 & 0.0415 & 0.0536 & $23 \%$ \\
\hline & & & NEQ & 468 & 0.0068 & 0.0300 & $77 \%$ \\
\hline \multirow{2}{*}{ c5315 } & \multirow{2}{*}{8220} & \multirow{2}{*}{6744} & EQ & 4858 & 0.4685 & 0.5764 & $19 \%$ \\
\hline & & & NEQ & 4807 & 0.0142 & 0.0000 & 0 \\
\hline \multirow{2}{*}{ C6288 } & \multirow{2}{*}{10992} & \multirow{2}{*}{11438} & EQ & 6791 & 2.8563 & 2.1655 & $-32 \%$ \\
\hline & & & NEQ & 6790 & 0.0178 & 0.0505 & $65 \%$ \\
\hline \multirow{2}{*}{ c7552 } & \multirow{2}{*}{8349} & \multirow{2}{*}{6267} & $\mathrm{EQ}$ & 3469 & 0.4462 & 0.8264 & $46 \%$ \\
\hline & & & NEQ & 3465 & 0.0162 & 0.0399 & $59 \%$ \\
\hline \multirow{2}{*}{ c880 } & \multirow{2}{*}{2110} & \multirow{2}{*}{1641} & EQ & 952 & 0.0730 & 0.1536 & $53 \%$ \\
\hline & & & NEQ & 880 & 0.0057 & 0.0207 & $72 \%$ \\
\hline
\end{tabular}

TABLE III. COMPARISON OF OUR LOGIC CHECKER AND ABC 
revised) of netlists with the same logic function. Then we insert some logical errors into the revised netlist by randomly changing the gate type. Besides, we compare our proposed method with $A B C$ 's combinational equivalence checking (CEC) method that is used in [7]. we use the $A B C$ tool [15] to perform the CEC of circuits, which have been removed splitters. $\mathrm{ABC}$ is used as a static library. We call the interface "Cmd_CommandExecute" to execute the command "cec golden.v revised.v", and record the runtime. The results are shown in TABLE III. The second and third columns represent the total number of logic gates in the golden netlist and the revised netlist, respectively. The fourth column represents the result of the logic checker. The fifth column represents the number of equivalent nodes merged. The sixth and seventh columns represent the verification time of our logic checker and ABC, respectively. Besides, we use the ANDF flip-flop to replace the bit-slice-based loops in bit-slice-based cyclic circuits and then use the Formality [16] to verify its logic function.

We compared ABC's CEC method with our proposed method which merged equivalent nodes and found that our proposed method reduces the runtime by $43 \%$. For the c6288 circuit, compared with $\mathrm{ABC}$, our program has worse results, which may be caused by the fewer logical cones of its network partition. Overall, our method has good performance.

\section{Conclusions}

In this paper, we propose the equivalence checking framework for superconducting RSFQ logic circuits, which is suitable for acyclic circuits and bit-slice-based cyclic circuits. For logic checker, we propose a network partition method with linear time complexity. The miter is simplified by removing equivalent nodes and divided into logic cones iteratively. The SMT solver is used to solve the linear equations constructed by the logic cone. The results clearly show the feasibility of our approach on the superconducting RSFQ benchmark. Compared with ABC's CEC method, our proposed method saves $43 \%$ of runtime on average. Due to the limitation of experimental data, the scale of the RSFQ logic circuits verified by our proposed approach is relatively small. Therefore, our next work will be to perform the equivalence checking of larger-scale superconducting RSFQ logic circuits. Besides, the clock network is very important for superconducting RSFQ logic circuits, there are some dual-clock circuits [17] that are similar to CMOS sequential circuits. For those circuits, we also focus on their equivalence check in further.

\section{ACKNOWLEDGMENTS}

This work was supported in part by the Strategic Priority Research Program of Chinese Academy of Sciences under Grant XDA18000000; and in part by the National Natural Science Foundation of China under Grant 61732018, 61872335, 61802367.

\section{REFERENCES}

[1] G. M. M. National Security Agency/Central Security Service Fort George, Superconducting Technology Assessment: Defense Technical Information Center, 2005.

[2] R. Sato, Y. Hatanaka, Y. Ando, M. Tanaka, A. Fujimaki, K. Takagi, and N. Takagi, "High-Speed Operation of Random-Access-Memory-Embedded Microprocessor With Minimal Instruction Set Architecture Based on Rapid Single-Flux-Quantum Logic," IEEE Transactions on Applied Superconductivity, vol. 27 , no. 4 , pp. 1-5, 2017.

[3] W. Chen, A. V. Rylyakov, V. Patel, J. E. Lukens, and K. K. Likharev, "Rapid single flux quantum T-flip flop operating up to $770 \mathrm{GHz}$," IEEE Transactions on Applied Superconductivity, vol. 9, no. 2, pp. 3212-3215, 1999.

[4] K. K. Likharev, and V. K. Semenov, "RSFQ logic/memory family: a new Josephson-junction technology for sub-terahertz-clock-frequency digital systems," IEEE Transactions on Applied Superconductivity, vol. 1, no. 1, pp. 328, 1991.

[5] J. A. Delport, K. Jackman, P. 1. Roux, and C. J. Fourie, "JoSIM-Superconductor SPICE Simulator," IEEE Transactions on Applied Superconductivity, vol. 29, no. 5, pp. 1-5, 2019

[6] A. D. Wong, K. Su, H. Sun, A. Fayyazi, M. Pedram, and S. Nazarian, "VeriSFQ A Semi-formal Verification Framework and Benchmark for Single Flux Quantum Technology," 2019.

[7] A. Fayyazi, S. Nazarian, and M. Pedram, "qEC: A Logical Equivalence Checking Framework Targeting SFQ Superconducting Circuits.” pp. 1-3.

[8] G.-m. Tang, "Studies on Datapath Circuits for Superconductor Bit-Slice Microprocessors.," Kyoto University, 2016.

[9] G. Tang, P. Qu, X. Ye, and D. Fan, "Logic Design of a 16-bit Bit-Slice Arithmetic Logic Unit for 32-/64-bit RSFQ Microprocessors," IEEE Transactions on Applied Superconductivity, vol. 28, no. 4, pp. 1-5, 2018.

[10] G. Tang, K. Takata, M. Tanaka, A. Fujimaki, K. Takagi, and N. Takagi, "4-bit Bit-Slice Arithmetic Logic Unit for 32-bit RSFQ Microprocessors," IEEE Transactions on Applied Superconductivity, vol. 26, no. 1, pp. 1-6, 2016.

[11] G. Pasandi, A. Shafaei, and M. Pedram, "SFQmap: A Technology Mapping Tool for Single Flux Quantum Logic Circuits.” pp. 1-5.

[12] A. Niemetz, M. Preiner, and A. Biere, "Boolector 2.0," 7. Satisf. Boolean Model. Comput., vol. 9, pp. 53--58, 2014.

[13] F. Brglez, and H. Fujiwara, "A Neutral Netlist of 10 Combinational Benchmark Circuits and a Target Translator in Fortran." pp. 677-692.

[14] R. Fu, Z.-M. Zhang, G.-M. Tang, J. Huang, X.-C. Ye, D.-R. Fan, and N.-H. Sun, "Design Automation Methodology from RTL to Gate-level Netlist and Schematic for RSFQ Logic Circuits," in Proceedings of the 2020 on Great Lakes Symposium on VLSI, Virtual Event, China, 2020, pp. 145-150.

[15] R. Brayton, and A. Mishchenko, "ABC: An Academic Industrial-Strength Verification Tool." pp. 24-40.

[16] Synopsys. "Formality," 2, 2021; https://www.synopsys.com/implementationand-signoff/signoff/formality-equivalence-checking.html.

[17] G. Pasandi, and M. Pedram, "An Efficient Pipelined Architecture for Superconducting Single Flux Quantum Logic Circuits Utilizing Dual Clocks,” IEEE Transactions on Applied Superconductivity, pp. 1-1, 2019. 